Gut, 1987, 28, 1467-1473

\title{
An epidemic of pseudomembranous colitis: Importance of person to person spread
}

\author{
N P M NOLAN, C P KELLY, J F H HUMPHREYS, C COONEY, \\ R O'CONNOR, T N WALSH, D G WEIR, AND D S O'BRIAIN
}

From the Departments of Histopathology, Clinical Microbiology and Gastroenterology, St James' Hospital, Dublin, Trinity College Medical School, Dublin, and Department of Medicine, Royal City of Dublin Hospital, Dublin, Ireland

SUMmary Twenty three cases of pseudomembranous colitis (PMC) occurred in three hospitals in 10 months. Retrospective analysis shows that they represented a single epidemic with a readily traceable chain of person-to-person contact within and between hospitals. Most patients had severe pre-existing illness and all had broad spectrum antibiotics, including either ampicillin/ amoxycillin, a broad spectrum cephalosporin (particularly cefotaxime), or both. All patients had severe diarrhoea and all responded to vancomycin, but relapse occurred in five. Ten patients eventually died, principally because of underlying disease rather than from PMC. Failure to find fibrin thrombi in blood vessels in biopsies and the scanty distribution of non-invasive bacteria supports the concept of mucosal damage by bacterial toxin, rather than by direct infection, or ischaemia. Although environmental colonisation cannot be excluded, the observed pattern of spread suggests a major role for direct person-to-person crossinfection in the spread of disease in this outbreak.

Pseudomembranous colitis (PMC) is an inflammatory condition of the colon, usually occurring after broad spectrum antibiotic therapy, and associated with colonisation by toxigenic Clostridium difficile. We report an epidemic of PMC in three hospitals of our hospital group, in which there was temporal and geographical clustering of cases suggestive of crossinfection. C difficile is generally considered an opportunistic pathogen which may cause PMC in patients who have received broad spectrum antibiotics. This outbreak, taken in conjunction with other recent reports of case clustering, ${ }^{1-3}$ emphasises the role of cross infection in the pathogenesis of PMC.

\section{Methods}

PATIENTS

An unusual number of cases of PMC occurred in hospital 1 under the care of various clinicians but predominantly in two wards. Initial review of these cases led to the finding that an undue number of cases

Address for correspondence: Dr Niamh Nolan. Department of Histopathology. St James" I Iospital. Dublin 8 . Ireland.

Received for publication 15 April 1987 had also been identified in hospital 2 . A retrospective study was started. Clinicians in all seven hospitals of the group were questioned (in particular all endoscopists) about cases of PMC occurring in the previous year. Histopathological surgical and autopsy records for the previous year were reviewed for cases diagnosed as PMC and all microbiological isolates of $C$ difficile were recorded. Hospital charts of all patients identified by these procedures were reviewed and those not having a clinical illness consistent with PMC were excluded. In addition to 15 cases initially known to the clinicians at hospitals 1 and 2 a further eight cases were identified by this screening procedure. Twenty patients in whose faeces $C$ difficile was cultured showed no clinical evidence of PMC and these, regarded as asymptomatic colonisation, were eliminated from the study. We feel it unlikely that any diagnosed cases of PMC within our hospital group were not included in the study.

After these investigations it was found that the cases of PMC were limited to three hospitals, all of which are members of a larger hospital group, served by a central laboratory. Each affected hospital has 
approximately 200 beds and caters for general medical and surgical patients as well as selected specialities. In the other four general hospitals of the group representing 2000 beds no confirmed, and only two possible cases of PMC were identified during the study period.

Patients were diagnosed as suffering from PMC when a suggestive clinical presentation was supported by endoscopic and histological findings and/or positive microbiology. Clinical presentation entailed the development of severe diarrhoea during or after a course of broad spectrum antibiotic therapy.

Samples of faeces were inoculated onto Clostridium difficile selective medium, CCFA (d-cycloserine $0.5 \mathrm{~g} / \mathrm{l}$, cefoxitin $0.01 \mathrm{~g} / \mathrm{l}$ and fructose agar). ${ }^{+}$Faeces were cultured undiluted and at $1 / 100$ and $1 / 1000$ dilutions in saline and incubated in an anaerobic cabinet for at least three days at $37^{\circ} \mathrm{C}$. Isolates were identified as $C$ difficile on the basis of colonial morphology, chartreuse fluorescence under ultraviolet light and a typical gas liquid chromatography trace. The evaluation of cytotoxin which is not assessed routinely in our laboratory was not available retrospectively. Toxin production was identified in the only case (no 10) from which an isolate was available. Faeces were also cultured on desoxycholate citrate agar and a campylobacter selective medium" to exclude salmonella, shigella, and campylobacter infections as a cause of symptoms. In addition samples were examined for the presence of ova and parasites.

In most cases colonoscopy or sigmoidoscopy (with colonic mucosal biopsy) was also carried out. The typical features of PMC are the presence of multiple yellow plaques or pseudomembranes $(0 \cdot 2-2 \mathrm{~cm}$ in size

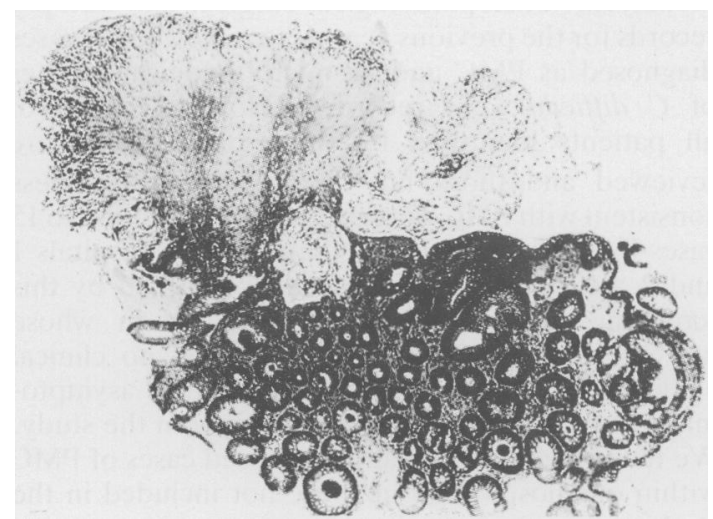

Fig. 1 Pseudomembranous colitis - the T2 pathognomonic lesion. Mushroom shaped exudate of fibrin, polymorphs and mucus originates from a distended gland and sprays onto the luminal surface of the mucosa to form the pseudomembrane seen at endoscopy. on the mucosal surface. The intervening mucosa is normal, but in more severe cases, the plaques may coalesce.

Haematoxylin and eosin stained histological sections were classified by the system of Price and Davies? into types 1-3. The type 2 (T2) lesion, considered pathognomonic, shows distended glands with 'mushrooming' from these of an exudate consisting of mucin, epithelial cell debris, polymorphs and fibrin onto the luminal aspect of the bowel (Fig. 1). The earliest lesion is the type 1 (T1), consisting of a luminal spray of similar exudate which originates in the lamina propria. The most severe form (T3) is complete mucosal necrosis indistinguishable from ischaemic injury. Histopathological sections were also stained by phosphotungstic acid haematoxylin (PTAH) for the presence of fibrin thrombi, and with the Brown Hopps Gram stain to identify bacteria. Mucin was identified using the periodic acid Schiff (PAS) and Alcian blue methods.

\section{Results}

Relevant features of the 23 patients involved in the outbreak are summarised in the Table. Fourteen were men, and nine women. The age range was 38 to 85 years (mean age 65.7 years). As shown in the Table, patients were admitted for a variety of reasons with exacerbation of chronic obstructive airways disease being the most common. The length of hospital stay varied from three to 72 weeks, the mean being 11 weeks. Seventy four per cent of patients spent more than six weeks in hospital.

The most common indication for antibiotics was for treatment of a lower respiratory tract infection ( 16 cases), four for surgical prophylaxis and one each for pericaecal abscess, urinary tract infection and burns. The duration of antibiotic therapy ranged from four days to one month, with an average of 14 days. Pseudomembranous colitis presented in all cases with diarrhoea which was usually severe and incapacitating and occurred from three days after beginning a course of antibiotic therapy to one month after completing the course: the mean date of onset of symptoms was 14 days from the initiation of antibiotic therapy. Figure 2 shows the time of onset of symptoms (arrow) related to the course of the hospital stay of each patient (solid horizontal line). In most cases symptoms first occurred in hospital, however, in two cases, (13 and 14) symptoms occurred at home, but in both instances after discharge from an affected ward in hospital 2. The antibiotics most commonly used were ampicillin/amoxycillin (in 17 cases) and cefotaxime (10 cases).

Endoscopy with biopsy was done in 16 of the 23 patients and features which were considered to be 
Table Clinical and diagnostic features of cases

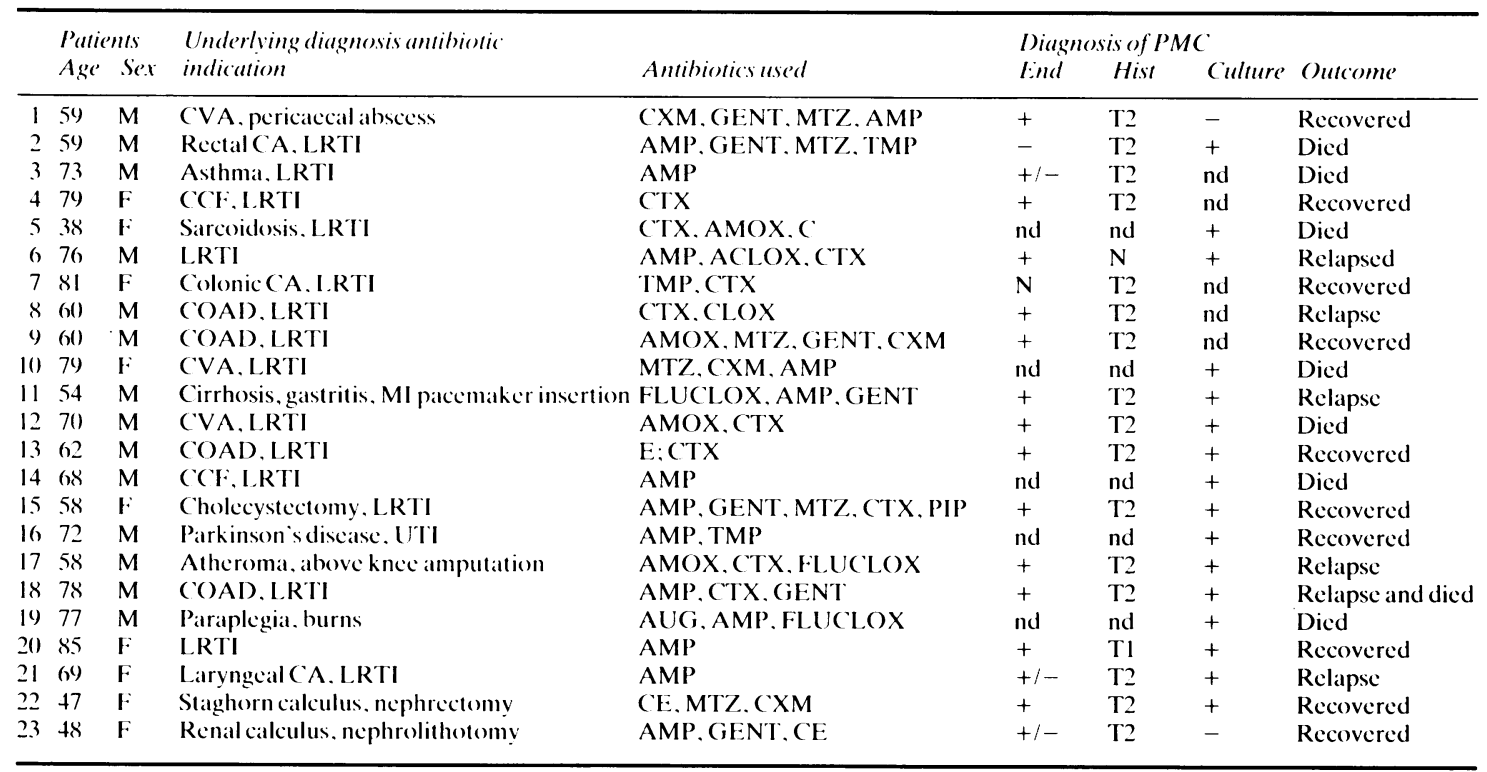

END = endoscopy $+=$ diagnostic of PMC $;+/-=$ abnormal but not diagnostic of PMC: $N=$ normal; nd = not done; Hist =histology; $\mathrm{T} 1, \mathrm{~T} 2=$ sec text $; \mathrm{CVA}=$ cerebrovascular accident $: \mathrm{LRTI}=$ lower respiratory tract infection; $\mathrm{CXM}=$ cefuroxime; $\mathrm{GENT}=\mathrm{gentamicin}$; $\mathrm{AMP}=$ ampicillin; $\mathrm{MTZ}=$ metronidazole; $\mathrm{TMP}=$ trimethoprim $; \mathrm{CTX}=$ cefotaxime; $\mathrm{AMOX}=$ amoxycillin; $\mathrm{COAD}=$ chronic obstructive airways disease; $\mathrm{CCF}=$ congestive cardiac failure; $\mathrm{C}=$ ehloramphenicol; $\mathrm{E}=$ erythromycin; $\mathrm{CE}=$ cephradine; $\mathrm{AUG}=$ augmentin: ACLOX =ampliclox; FLUCLOX =flucloxicillin.

diagnostic of PMC were seen in $12(75 \%)$. Nondiagnostic abnormalities were seen in three cases. One case which was considered endoscopically normal showed pathognomonic histological lesions. Two further patients had typical features of PMC in the colon at autopsy. Faecal culture for $C$ difficile was carried out in 18 patients and the organism successfully isolated in all but two of these $(89 \%)$. Because of the retrospective nature of this study toxin identification was undertaken in only one case, where it was positive. No other pathogens apart from $C$ difficile were isolated from patients with diarrhoea in this series.

\section{HISTOLOGY}

Sixteen of $18(89 \%)$ patients where histological examination was done showed diagnostic (T2) lesions of PMC. Two of these were from autopsy cases, the remainder had undergone endoscopy with biopsy. Histology identified PMC in the three patients who had non-diagnostic endoscopic abnormalities and in one case reported as normal.

Biopsies were taken from a single abnormal area in some cases, and as 'serial' biopsies from different sites in the colon in others. Overall $45 \%$ of biopsy fragments showed diagnostic lesions (multiple levels were cut). The majority of the remainder were normal or showed only minor changes. In one case (no 6) a single biopsy taken from mucosa with a typical endoscopic appearance was normal. In three other cases only one of eight, four and four biopsies respectively were positive. Forty per cent of the biopsies obtained from the transverse colon and $31 \%$ from the rectum yielded $\mathrm{T} 2$ lesions, as compared with $21 \%$ and $20 \%$ from the sigmoid and descending colon.

Each block was stained by PTAH to identify fibrin thrombi in vessels. Thrombi were not identified in any of the 18 cases, either close to, or removed from the lesion of PMC. Mucin depletion was shown in the glands adjacent to the PMC lesions. A Gram stain identified scanty Gram positive and negative bacteria within the exudate in many cases but invasive organisms were not identified within the epithelium.

In five of the 23 patients the diagnosis of PMC was not based on pathognomonic endoscopic or histological features. Diagnosis in these five is based on the development of severe incapacitating diarrhoea during or after broad spectrum antibiotic therapy, growth of $C$ difficile from stool and marked symptomatic improvement within 48 hours of introduction of treatment with oral vancomycin. Diagnosis based 
on these factors is not absolute, but we felt it justified to include these patients within the context of an outbreak of PMC and in the absence of other causes.

OUTCOME

The duration of PMC was difficult to ascertain precisely from a retrospective review of the records but appeared to last about five days on average. All patients showed response to oral vancomycin therapy. Nine recovered completely on this treat- ment. Ten patients died later as a result of their primary disease. Five patients relapsed, as diagnosed by recurrence of symptoms after a course of vancomycin. In the two of these recurrences where it was sought, $C$ difficile was cultured. One of these five patients was among those who subsequently died. The other four responded to oral vancomycin therapy. Barrier nursing was introduced where possible. There was no difference in the standard of barrier nursing applied to the patients who relapsed.

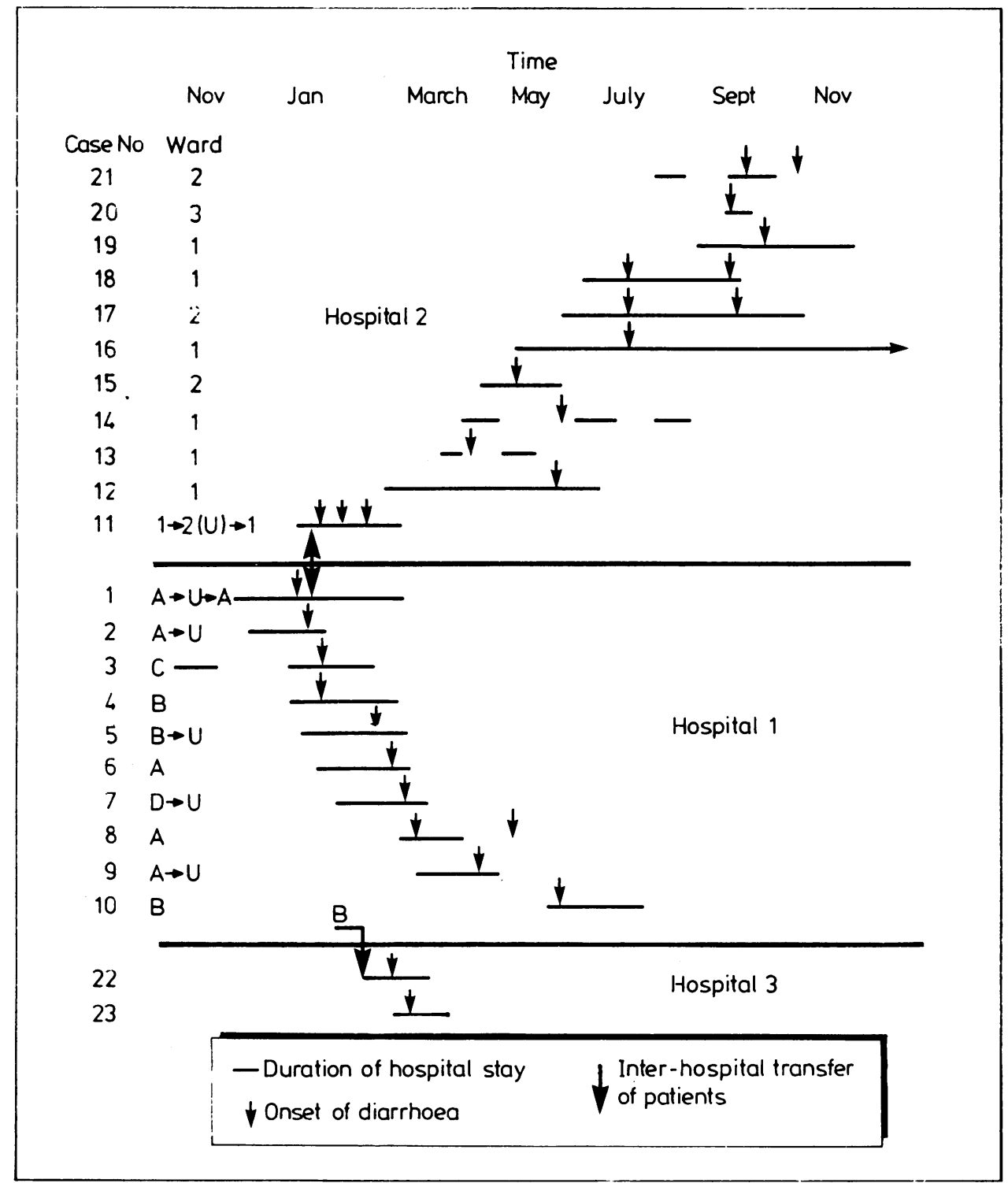

Fig. 2 Cases of PMC in all three hospitals, 1, 2, and 3. The duration of hospital stay, the temporal and geographical relationship of cases and the transfers of patient 11 to hospital 1 and patient 22 to hospital 3 are represented. 
In two cases (nos 7 and 21 ) relapse occurred at home after discharge from hospital.

\section{EPIDEMIOLOGICAL DATA}

Twenty three patients were diagnosed as suffering from PMC over a 10 month period. This represents a dramatic increase above the usual number of cases observed which we estimate as approximately three per year in our hospital group. Cases occurred in three separate hospitals but showed marked temporal clustering (Fig. 2). Within each hospital geographical clustering was also observed. Examination of patient transfers reveals a likely mode of spread of the outbreak from one hospital to the other two.

The earliest case occurred in hospital 1 where nine other cases followed over a five month period. The outbreak in this hospital was largely confined to two wards, $A$ and $B$ where eight of the 10 patients were cared for. Four of these patients had also spent a considerable length of time in the intensive care unit (U) and it was in this unit that the index case occurred. A ninth case, nursed in a separate ward, D, had also been in the intensive care unit before developing symptoms and the last case was nursed in an unrelated ward. Eleven cases occurred in hospital 2 and again geographical clustering was evident in that all but one patient (no 20) was nursed in one of the two affected wards (wards 1 and 2). The time scale of the outbreak in this hospital was more protracted, spanning nine months. By examining patient records it was found that there was extensive overlap in the period of inpatient treatment of the affected patients. Although their symptoms began at home, previous hospitalisation in an affected ward provides the link for cases 13 and 14 . Two cases, one of whom was transferred from hospital 1, ward B, were diagnosed in hospital 3 . Both patients were nursed in the same ward, in adjoining beds, and at the same time.

There is an obvious temporal relationship between the outbreaks of PMC in the three hospitals (Fig. 2). Examination of the movements of patients between the hospitals provided further evidence in favour of a single source for the outbreak. The first affected patient in hospital 2 (case no 11, Fig. 2) was admitted with an acute myocardial infarction and complete heart block. This required temporary transfer to hospital 1 for insertion of a permanent cardiac pacemaker under flucloxacillin prophylaxis. While staying in hospital 1, he had been nursed in the intensive care unit (U) where the first affected patient in hospital 1 was being treated at the time. He developed severe diarrhoea four days after his return to hospital 2. Similarly the first of the two affected patients in hospital 3 had recently been transferred from hospital 1, ward B. In conclusion, the outbreak would appear to have begun in the intensive care unit of hospital 1 and have been transferred to hospitals 2 and 3 by patients no 11 and 22 (Fig. 2) respectively. (Some of these cases were referred to in advisory notes on antibiotic association. (Lancet 1985, ii: 846():888 and Lancet 1986; i: 8472:102-3.))

\section{Discussion}

Pseudomembranous colitis was first described by Finney in 1893. " A marked increased incidence in the 1960 's led to the recognition of prior antibiotic therapy as a major aetiological factor. It was not until 1978, however, that toxigenic $C$ difficile was identified as a causative agent in antibiotic associated PMC." "The relative importance of exogenous and endogenous sources of infection in the pathogenesis of $C^{\prime}$ difficile associated colitis remains controversial. ${ }^{11-13} C$ difficile may be cultured from a proportion of asymptomatic patients - $3 \%,{ }^{14}$ but few of these develop symptoms even if they also are given broad spectrum antibiotics. Consequently it is felt that the expense and disruption to ward routine involved in the detection and treatment of healthy carriers is not justified..$^{\text {is }}$ Such carriers were not sought in this outbreak and we feel it unlikely that they played a role in the spread of disease as a chain of symptomatic infected patients was so readily discovered.

A notable feature of this series is that the majority of patients were elderly and that all were suffering from debilitating illnesses, many of which were chronic. These factors are reflected in the mean duration of hospital stay, which was 11 weeks. Mortality rates in PMC have been reduced by the introduction of specific therapy with oral vancomycin. ${ }^{171}$ There was, however, a high mortality rate in our series $-43.5 \%$ which largely reflects the debilitated state of the affected patients rather than the severity of their PMC. In no case was PMC considered to be the principal cause of death and in only three was the condition active at the time of death.

All patients in our series received ampicillin/ amoxycillin (18 cases) and/or a broad spectrum cephalosporin, mostly cefotaxime $(10$ cases $)$. Since antibiotic related PMC was first described in relation to clindamycin a large number of antibiotics have been implicated, but with combination antimicrobial chemotherapy as in this and other modern series, it is difficult to implicate a single antibiotic. Ampicillin which is prominent in our series, however, has been repeatedly associated with PMC. Third generation cephalosporins have also been increasingly implicated in PMC. ${ }^{11}$ Cefotaxime, a third generation cephalosporin has been reported to 
have a minimal association with $\mathrm{PMC}^{2021}$ and it has been suggested that this may be explained by the minimal effect of the drug on intestinal flora,"2 thereby preventing colonisation by $C$ difficile. Therefore the prominence of cefotaxime in this series is striking. There is also recent evidence to suggest that the cephalosporins, especially cefotaxime, are more frequently associated with PMC than prescribing frequency alone would indicate..$^{23}$ While ampicillin/ amoxycillin and cefotaxime were extensively prescribed in the affected hospitals during the period of the outbreak, they were also widely used in the other group hospitals which remained free of PMC.

There have been previous reports of case clustering $^{1-3}$ which, together with this series suggest that crossinfection may have a significant role in the pathogenesis of PMC. $C$ difficile is a spore forming organism whose inactive spores may remain for some time as environmental contaminants before infecting a susceptible host. Such environmental contamination has been shown to occur in association with cases of PMC and other authors have suggested that this may provide a route for $C$ difficile infection. ${ }^{112}$ In this series, however, person-to-person spread is a more likely route. Although studied several months later a clear chain of person-to-person contact could be established between almost all the cases. This was facilitated by the long hospital stay of these chronically ill patients. All patients, with the exception of cases 3 and 20, shared a ward and/or toilet facilities with another patient. The pattern of spread from hospital 1 to hospital 2 and 3 is consistent with a person to person route of crossinfection and is difficult to explain by environmental contamination by spores. Once the chain of cases was broken by treating patients with vancomycin, barrier nursing and discharge, no further cases occurred in hospitals 1 and 3 suggesting that environmental contamination by spores was not a factor in this outbreak. In hospital 2 , however, the problem was much more difficult to eradicate, despite barrier nursing and vancomycin therapy. Many of the affected patients in this hospital had a particularly long duration of hospital stay (median 28 weeks). We feel that the continuing presence of 'infected' individuals, rather than environmental contamination may have been the key factor explaining the persistence of the outbreak in this hospital. Other workers have recently shown $C$ difficile to be endemic among elderly patients in chronic care wards, and have commented on the difficulties encountered in eradicating the bacterium by normal isolation methods. ${ }^{2+}$ Environmental contamination as a factor in the persistence of the epidemic in hospital 2 is unlikely, but cannot be entirely excluded. $C$ difficile which is a ubiquitous contaminant was not sought from the environment during the outbreak. In contrast with the investigation of environmental contaminating pathogens such as staphylococcus and pseudomonas where specific typing systems are crucial, there is no readily available typing system for $C$ difficile so that the pathogenic significance of its finding in the environment is difficult to assess; conversely the failure to find the bacterium in the environment does not exclude a focus of contamination.

The material collected in this study shows the value of multiple colonic biopsies in the identification of PMC histologically, as the disease is often patchy in its distribution. Rectal involvement was prominent, both from biopsy and autopsy material, a finding which contrasts with the rectal sparing described by Tedesco. ${ }^{.5}$ The lack of fibrin thrombi in blood vessels and the lack of invasive bacteria provide histological support for the concept that PMC is caused by the toxins produced by $C$ difficile, rather than by ischaemia or by direct infection. This seems to indicate that the microthrombi identified in the early cases of PMC, 7 which were often studied at autopsy, were an effect, rather than a cause of the disease.

This report describes outbreaks of PMC in three hospitals which represent a single epidemic. Outbreaks occurred simultaneously and there was marked geographical clustering within each hospital. Interhospital transfer of patients provides the probable route of spread of the disease. The lack of a reliable $C$ difficile typing system and the retrospective nature of this study make conclusive proof impossible. It is not clear why this outbreak should have occurred at this time. The prominence of severe respiratory disease in many of the patients raises the possibility that a combination of hypoxia and change in local immunity may be factors in allowing the production of the toxin from the anaerobic $C$ difficile. This, together with the possible presence of a particularly virulent strain of $C$ difficile, pattern of antibiotic usage, long stay of patients and especially person-toperson spread of disease, may all have contributed to the occurrence of the outbreak.

\section{References}

1 Milligan DW, Kelly JK. Pseudomembranous colitis in a leukaemia unit, a report of five fatal cases. J Clin Pathol 1979; 32: 1237-43.

2 Bennett CC, Allen E, Millard PH. Clostridium difficile diarrhoea; a highly infectious organism. Age Ageing 1984; 13: 363-6.

3 Greenfield C, Burroughs A, Szawathowski M, Bass N, Noone P, Pounder R. Is pseudomembranous colitis infectious? Lancet 1981; i: 371-2.

4 George WL, Sutter VL, Citron D, Finegold SM. Selective and differential medium for the isolation of Clostridium difficile. J Clin Microbiol 1976; 9: 214-9. 
5 Berg JD, Mills RG, Coleman DJ. Improved gas-liquid chromatography method for the identification of Clostridium difficile. J Clin Pathol 1985; 38: 108-13.

6 Skirrow MB. Campylobacter enteritis: a "new" disease. Br Med J 1977; 2: 9-11.

7 Price AB, Davies DR. Pseudomembranous colitis. J Clin Pathol 1977; 30: 1-12.

8 Finney JMT. Gastroenterostomy for cicatrising ulcer of the pylorus. Bull Johns Hopkins Hosp 1893; 4: 53-5.

9 Larson HE, Parry JV, Price AB, Davies DR, Dolby J, Tyrell DAJ. Undescribed toxin in pseudomembranous colitis. Br Med J 1977; 1: 1246-8.

10 Bartlett JG, Chang TW, Gurwith M, Gorbach SL, Onderdank AB. Antibiotic-associated pseudomembranous colitis due to toxin producing clostridium. $N$ Engl J Med 1978; 298: 531-4.

11 Malamou-Ladas H, O’Farrell S, Nash JQ, Tabagahali S. Isolation of Clostridium difficile from patients and the environment of hospital wards. J Clin Pathol 1983; 36: $88-92$.

$12 \mathrm{Kim} \mathrm{KH}$, Fekety R, Batts $\mathrm{DH}$, et al. Isolation of Clostridium difficile from the environment and contacts of patients with antibiotic-associated colitis. $J$ Infect Dis 1981; 143: 42-50.

13 Mulligan ME, George WL, Rolfe RD, Finegold SM. Epidemiological aspects of Clostridium difficile induced diarrhoea and colitis. Am J Clin Nutr 1980; 33: 2533-8.

14 George WL, Sutter VL, Finegold SM. Antibiotic agent induced diarrhoea - a bacterial disease. J Infect Dis 1977; 13: 822-8.
15 George RH. The carrier state: Clostridium difficile. $J$ Antimicrob Chemother 1986; 18: suppl A: 47-58.

16 Hall SM, Calver GP. Williams M. A hospital outbreak of Clostridium difficile? J Hosp Infect 1985; 6: 312-22.

17 Tedesco F, Markham R. Gurwith M. Christic D. Bartlett JG. Oral vancomycin for antibiotic-associated pseudomembranous colitis. Lancel 1978; ii: 226-8.

18 Tedesco FJ. Pseudomembranous colitis: Pathogenesis and Therapy. Med Clin North Am 1982; 66: 65.5-64.

19 Miller SD, Blake M. Miliotis M. Still C, Taubin A. Koornhof HJ. Antibiotic-associated diarrhoca and pseudomembranous colitis caused by Clostridium difficile. SA Med J 1983; 63: 936-9.

20 Parker RH, Park SY. Safety of cefotaxime and other new B-Lactam antibiotics. J Antimicrob (hemother 1984; suppl B: 331-5.

21 Carmine AA. Broghden RN, Heel RC, Speight TM, Avary GS. Cefotaxime, A review of its antibacterial activity, pharmacological properties and therapeutic use. Drugs 1983; 25: 223-89.

22 Sunakawa K, Akita H, Iwata S, Sat Y. The influence of cefotaxime on intestinal flora and bleeding diathesis in infants and neonates compared with other B-Lactams. J Antimicrob Chemother 1984; suppl B: 317-24.

23 Slack RCB, Finch RG. Pseudomembranous colitis. [Letter]. Lancet 1985; ii: 1358-9.

24 Bender BS, Bennett R, Laughan B, et al. Is Clostridium difficile endemic in chronic-care facilities? Lancet 1986; ii: 11-3.

25 Tedesco FJ. Antibiotic-associated pseudomembranous colitis with negative proctosigmoidoscopy examination. Gastroenterology 1979; 77: 295-7. 